\title{
Cladoceran birth and death rates estimates: experimental comparisons of egg-ratio methods
}

\author{
WILFRIED GABRIEL, BARBARA E. TAYLOR* and \\ SUSANNE KIRSCH-PROKOSCH Department of Physiological Ecology, \\ Max Planck Institute for Limnology, Plön, West Germany
}

SUMMARY. 1. Birth and death rates of natural cladoceran populations cannot be measured directly. Estimates of these population parameters must be calculated using methods that make assumptions about the form of population growth. These methods generally assume that the population has a stable age distribution.

2. To assess the effect of variable age distributions, we tested six egg ratio methods for estimating birth and death rates with data from thirty-seven laboratory populations of Daphnia pulicaria. The populations were grown under constant conditions, but the initial age distributions and egg ratios of the populations varied. Actual death rates were virtually zero, so the difference between the estimated and actual death rates measured the error in both birth and death rate estimates.

3. The results demonstrate that unstable population structures may produce large errors in the birth and death rates estimated by any of these methods. Among the methods tested, Taylor and Slatkin's formula and Paloheimo's formula were most reliable for the experimental data.

4. Further analyses of three of the methods were made using computer simulations of growth of age-structured populations with initially unstable age distributions. These analyses show that the time interval between sampling strongly influences the reliability of birth and death rate estimates. At a sampling interval of 2.5 days (equal to the duration of the egg stage), Paloheimo's formula was most accurate. At longer intervals (7.5-10 days), Taylor and Slatkin's formula which includes information on population structure was most accurate.

\section{Introduction}

To study the factors controlling zooplankton population dynamics, it is helpful to obtain

*Present address: Savannah River Ecology Laboratory, Drawer E, Aiken, South Carolina 29801, U.S.A. information about demographic processes such as birth, recruitment and death. For example, correlations between estimated death rates of

Correspondence: Dr W. Gabriel, Department of Physiological Ecology, Max Planck Institute for Limnology, Postfach 165, D-2320 Plön, West Germany. 
the prey population and abundances of predatory species have been used to infer which predators are most important to the prey population (Hall, 1964; Wright, 1965). Birth and death rates of natural zooplankton populations cannot be measured directly. Instead, they are estimated from mathematical models of population growth. During the last 30 years, various methods for estimating birth and death rates have been developed. In this paper we examine estimators based on the egg ratio (the ratio of eggs to animals in the population; Edmondson, 1960), and compare their sensitivities to departure from the stable age distribution assumed by these methods.

These estimators are derived from models for exponential population growth. The birth rate $b$ at sampling time $t_{1}$ is estimated from the egg ratio by making assumptions about the age distribution of the eggs. The population growth rate $r$ is estimated from the change in population size from sampling time $t_{1}$, to sampling time $t_{2}$, which measures the average growth rate during the sampling interval $t_{2}-t_{1}$. The death rate $d$ is estimated by the difference between the birth rate and the population growth rate; this calculation assumes that the estimated birth rate applies for the entire interval between samples.

Natural processes cause the rates and forms of population growth to vary, so that age distributions of natural zooplankton populations are unlikely to remain stable, especially when environmental conditions are changing. To test whether departures from a stable age distribution affect the accuracy of birth and death rate estimates based on egg ratios, we compared estimated and actual death rates calculated with data from a set of thirty-seven laboratory populations of Daphnia pulicaria Forbes. We compared death rates because this estimate includes two important sources of error: error in the instantaneous estimate of the birth rate; and error in the extrapolation of the birth rate over the sampling interval. The populations were grown under constant conditions, but the initial age distributions and egg ratios were varied. Different initial distributions simulated effects of sudden environmental change on population structure. Six variations of the egg ratio method were applied to experimental data. The results show that all methods are affected by departures from the stable age distribution. A detailed insight into dynamics is difficult to gain solely by laboratory experiments. Therefore, we performed computer simulation as additional 'experiments' to study if and how the estimates depend on the sampling interval.

\section{The birth and death rate estimators}

Birth and death rate estimates based on the egg ratio were developed for short-lived animals with rapid, continuous reproduction and approximately exponential population growth. They are derived from quantities that can easily be measured for freshwater zooplankton such as cladocerans and rotifers, which usually carry their developing eggs. These methods have developed mostly independently of methods to estimate demographic parameters for longer-lived animals, such as fish (e.g. Ricker, 1975) and humans (e.g. Pollard, 1973; Keyfitz, 1977) and for populations that show strong cohort structure (applications to zooplankton populations include Rigler \& Cooley, 1974, Gehrs \& Robertson, 1975, and Hairston \& Twombly, 1985). Although methods for estimating secondary production of zooplankton rely on similar assumptions, they are also separate from work on population processes (see Winberg, 1971, and Edmondson \& Winberg, 1971).

The egg ratio was first used to estimate parameters of exponential population growth by Edmondson (1960), although Elster (1954) calculated a renewal coefficient ('Erneuerungs koeffizient') for the population from the egg ratio and egg development period. Edmondson's method was elaborated and refined by Edmondson (1968), Caswell (1972), Paloheimo (1974) and Argentesi, Bernardi \& di Cola (1974); and a similar method was developed in Smith's appendix to Cooper (1965). The accuracy and precision of these methods have since received considerable attention and development (Prepas \& Rigler, 1978; Seitz, 1979; Threlkeld, 1979; Polishchuk, 1980, 1982; DeMott, 1980; Keen \& Nassar, 1981; Polishchuk \& Ghilarov, 1981; Taylor \& Slatkin, 1981; Lynch, 1982, 1983; Dorazio \& Lehman, 1983).

The six methods that we compared are listed in Table 1. All assume population growth is 
TABLE 1. Formulae for estimating birth rates. Variables are: $b=$ instantaneous per capita birth rate in days ${ }^{-1}$, with subscript to indicate estimation method; $r=$ instantaneous per capita population growth rate; $E=$ female eggs; $N=$ female animais; $J=$ juvenile female animals; $A=$ adult female animals; $D_{\mathrm{E}}=$ duration of egg stage in days; $D_{\mathrm{J}}=$ duration of juvenile stage in days; $B=$ finite daily per capita egg hatching rate which is equivalent to the number of female eggs hatching per female over a 1-day interval.

\begin{tabular}{lll}
\hline Method & Equation & Source
\end{tabular}

1. Edmondson: $b_{\mathrm{ED}}$

2. Caswell: $b_{\text {CAS }}$

3. Paloheimo: $b_{\mathrm{PAL}}$

4. Seitz: $b_{\text {SEI }}$

5. Taylor \& Slatkin: $b_{\mathrm{TS}}$

iteration of

$b_{\mathrm{ED}}=\ln (B+1)$
$B=E / N D_{\mathrm{E}}$

$b_{\mathrm{CAS}}=\frac{\mathrm{rB}}{e^{r}-1}$

$B=E / N D_{\mathrm{E}}$

$b_{\mathrm{PAL}}=\frac{\ln (E / N+1)}{D_{\mathrm{E}}}$

iteration of $b_{\mathrm{S}}=\frac{E}{N} \frac{1-e^{r_{\mathrm{E}}}}{r_{\mathrm{E}}} \frac{r}{e^{r}-1} \frac{r_{\mathrm{E}}+b_{\mathrm{S}}-r}{1-e^{\left(r_{\mathrm{E}}+b-r\right) D_{E}}}$

$E / A=e^{b_{T S} D_{1}}\left\{e^{b_{T S} D_{E}-1}\right\}$

$b_{\mathrm{TSmod}}=b_{\mathrm{TS}} e^{b_{\mathrm{TS}} D_{\mathrm{J}}} A / N$
Edmondson, 1960

Caswell, 1972

Paloheimo, 1974

Seitz, 1979

Taylor \& Slatkin 1981, equation 7

Taylor \& Slatkin 1981, equation 22 exponential, so that

$$
N\left(t_{2}\right)=N\left(t_{1}\right) e^{r\left(t_{2}-t_{1}\right)}
$$

where

$$
r=b-d .
$$

$N\left(t_{1}\right)$ and $N\left(t_{2}\right)$ are populations at times $t_{1}$ and $t_{2}$, and $r, b$ and $d$ are the instantaneous growth, birth and death rates of the population. A stable age distribution is required for exponential growth if birth or death rates vary with the animal's age. Both certainly vary with age for cladocerans. As discussed in detail by Seitz (1979), these methods also assume that the death rates among all age classes, including eggs, are equal. The methods differ in the measure of the egg ratio (compare methods 1 , 2 and 3 with 5 and 6 ) and in the mechanism used to convert the egg ratio to a birth rate (compare methods 1,2 and 3). Method 6 includes a factor intended to compensate partially for effects of departures from the stable age distribution, as measured by the proportion of adults, on the inferred egg age distribution. In addition to these six methods we also analysed our experiments with the formulas of Argentesi et al. Their model, however, implicitly assumes not only stable but also uniform age distribution in the compartments of eggs, juveniles and adults. Therefore, under our experimental conditions it was not surprising that the model of Argentesi et al. gave results by one order of magnitude worse than the six other models.

All of these methods estimate the hatching rate of eggs from the egg development time and population structure. Population structure is measured very simply by the egg ratio. For example, Edmondson (1960) 'finite' birth rate assumes a uniform age distribution of eggs, while Paloheimo (1974) and Taylor \& Slatkin (1981) assume that the distributions are the stable age distributions associated with the measured egg ratios. Threlkeld's (1979), Dorazio \& Lehman's (1983) and Rigler \& Downing's (1984) methods are based on direct measures of the egg age distribution. From the egg age distribution the proportion of egg hatching in the next instance can be calculated; this number, converted to a per capita measure, is the instantaneous birth rate. Estimating the birth rate thus assumes that the current egg distribution has been correctly inferred from the current population structure. A potentially serious problem with methods 5 and 6 is the determination of the juvenile developmental time which is quite sensitive to food concentrations at low food levels (Roma- 
novski, 1984). Estimating the death rate as the difference between the birth rate and the population growth rate assumes that the instantaneous birth rate estimated for this instant remains constant during the interval over which population growth was measured. When environmental conditions or the population structure are unstable, birth rates vary, and this extrapolation can produce large errors.

The error in the estimated death rate includes the sum of errors in the birth and population growth rates. Errors in the death rate estimate may occur if: (1) the population did not satisfy the assumptions of the method for estimating the birth rate at that point in time; (2) the birth rate varied during the sampling interval, so that the instantaneous estimate of the birth rate differed from the average birth rate over the sampling interval; (3) population densities or development times needed to calculate the population growth or birth rates were measured inaccurately; (4) net migration to or from the population occurred; (5) sampling errors can also be serious problems (DeMott, 1980; Keen \& Nassar, 1981).

\section{Experimental methods}

Stock cultures of an obligately parthenogenetic clone of Daphnia pulicaria Forbes were grown in 1.5 litre beakers under dim light. To obtain neonates, several large gravid females were isolated in the evening, and young (aged $0-15 \mathrm{~h}$ ) were collected the next morning and transferred to another beaker. This procedure was repeated on successive days until juveniles of a range of ages were available to begin the experiments.

Experimental Daphnia populations were grown in a flow-through culture system similar to that described by Muck \& Lampert (1980). Ten $250 \mathrm{ml}$ chambers were placed in a temperature-controlled water bath held at $18^{\circ} \mathrm{C}$. Culture medium dripped into each culture chamber at the top at 1.5 litre $\mathrm{d}^{-1}$ and flowed out through a Nitex screen at the bottom into an overflow system. The medium was membrane-filtered lake water enriched with $1.5 \mathrm{mgClitre}^{-1}$ of Scenedesmus acutus Meyen, which was grown in a chemostat culture, as described by Lampert (1975). Fresh culture medium was prepared daily. The food concentrations were high so that food was not a limiting factor in the experiments.

A total of thirty-seven experimental cultures were run. Initial populations were ten, eleven or twelve Daphnia of varied ages and fecundities. Initial proportions of juveniles $J / N$ $(J=$ juvenile female animals, $N=$ female animals) were $0.0,0.50,0.75$ and 0.91 . The average adult egg ratio $E / A$ ( $E=$ female eggs, $A=$ adult female animals) ranged from 2.8 to 15.3 . Juvenile populations contained an even distribution of animals aged 1-5 days.

Each experiment lasted 7 days. The experiments were terminated by fixing the population of each chamber in sugar-formalin (Haney \& Hall, 1973). All of the animals were counted and measured, and the egg stage (according to Threlkeld, 1979) was determined for ovigerous animals. The chambers were checked every day for dead animals.

The egg development time $D_{\mathrm{E}}$ was estimated to be 2.95 days; the juvenile development time $D_{\mathrm{J}}, 6.5$ days. The adult stage was defined by body length or the presence of eggs in the brood pouch. The smallest length at which $75 \%$ of the population carried eggs was used as the criterion for minimum adult size. Additional details on the experimental setup are given by Kirsch (1982).

\section{Computer simulations}

To get additional information on the role of the sampling interval for the shortcoming of different egg ratio methods, we simulated the growth of a population by an age-structured, discrete time model. The model contains the essential features of a cladoceran life history: distinct egg, juvenile and adult stages, incubation of the developing eggs by the adult, and iteroparous reproduction. (The term egg is used here to include egg and embryonic stages.) The population was divided into sixtyseven age classes of 0.5 day duration. The durations of the egg, juvenile and adult stages were 5,12 and 50 time steps, or $2.5,6$ and 25 days. The lengths of the stages were adjusted to ensure that the corresponding Leslie matrix was positively regular (Pollard, 1973), so that the age distribution of the population would become stable over time. An adult produced a total of ten broods, carrying each brood for the 
duration of the egg stage. Strictly parthenogenetic reproduction was assumed. The death rate was set to 0 for all age classes except the last.

The models of age-specific fecundity were used in the simulations: constant (brood size $C=5$ for all ten broods); and increasing (brood size $C_{\mathrm{i}}=5+5(i-1)$ for brood number $\left.i=1,10\right)$. Three initial age distributions were simulated: uniform $\left(N_{i}=1\right.$ for the juvenile through adult age classes $i=5,66)$; negatively skewed $\left(N_{i}=62-(i-5)\right.$ for $\left.i=5,66\right)$; and positively skewed $\left(N_{i}=1+(i-5)\right.$ for $\left.i=5,66\right)$. Initial populations in the egg age classes $i=0,4$ were calculated from the numbers and fecundities of the adult animals. Proportions of juveniles $\mathrm{J} / \mathrm{N}$ were: 0.19 for the uniform age distribution; 0.35 for the negatively skewed distibution; and 0.04 for the positively skewed distribution. At stable age distribution the proportions were 0.80 for constant fecundity and 0.86 for increasing fecundity.

Birth, death and population growth rates were calculated for each 0.5 day time step in the simulations. Birth rates were estimated according to Paloheimo's, Taylor and Slatkin's, and Taylor and Slatkin's modified formulae (methods 3,5 and 6 in Table 1). Population growth rates were calculated using equation 1 with time intervals of $0.5,2.5,5$, 7.5 and 10 days. Death rates were calculated using the three birth rate estimates and the five population growth rates in equation 2 .

\section{Results}

\section{Actual birth and death rates of the experimental populations}

In thirty-seven experiments with initial and final populations from 920 to over 10,000 animals, only eight dead animals were found. This number of deaths corresponds to an instantaneous death rate $d=0.0003$ per day, which is negligibly small. In the following analyses, we assume that the actual death rate for the experimental populations were zero. The death rate of eggs was estimated from the proportion of eggs with arrested development (stage I) in broods at stages III-V. Among 350 broods at stages III-V, 234 out of 6298 eggs were in stage I, giving a death rate of $3.7 \%$ during the egg stage, or an instantaneous death

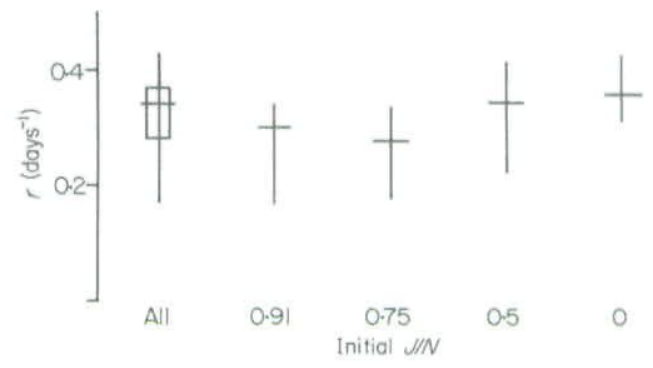

FIG. 1. Population growth rates $r$ for the experimental populations. Rates are shown for all experimental populations combined and for populations grouped by the initial proportion of juveniles $J / N$ ( $J=$ juvenile female animals; $N=$ female animals). Vertical bars show ranges of values; horizontal bars show medians. The box shows quartiles for the combined data.

rate of 0.013 per day. This estimate is conservative, because eggs which disintegrated or stopped developing after stage I were not detectable.

Population growth rates $r$ were calculated using equation 1 from the total numbers of animals at the beginning and end of the experiment. Because death rates were virtually zero, birth rates were equal to growth rates of the experimental populations. These birth and population growth rates ranged from 0.17 to 0.43 per day (Fig. 1). The rates were affected by the initial population structure. The treatments with low proportions of juvenile $(J / N$ $=0.0$ and $J / N=0.50$ ) tended to have higher birth rates.

\section{Changes in structure of the experimental populations}

Changes in the proportion of juvenile $J / N$ and the egg ratios $E / N$ and $E / A$ measure changes in the structure of these populations. (They may also measure response to changing environmental conditions, but that effect is negligible in these experiments, because the animals were preconditioned to the culture conditions.) For comparison with the experimental data, we estimated what the egg ratios and proportions of juveniles should be at the stable age distribution for this set of experimental populations according to these relations:

$$
\begin{aligned}
& E / N=e^{b D_{\mathrm{E}}}-1 \\
& E / A=e^{b D_{5}}\left(e^{b D_{\mathrm{E}}}-1\right)
\end{aligned}
$$



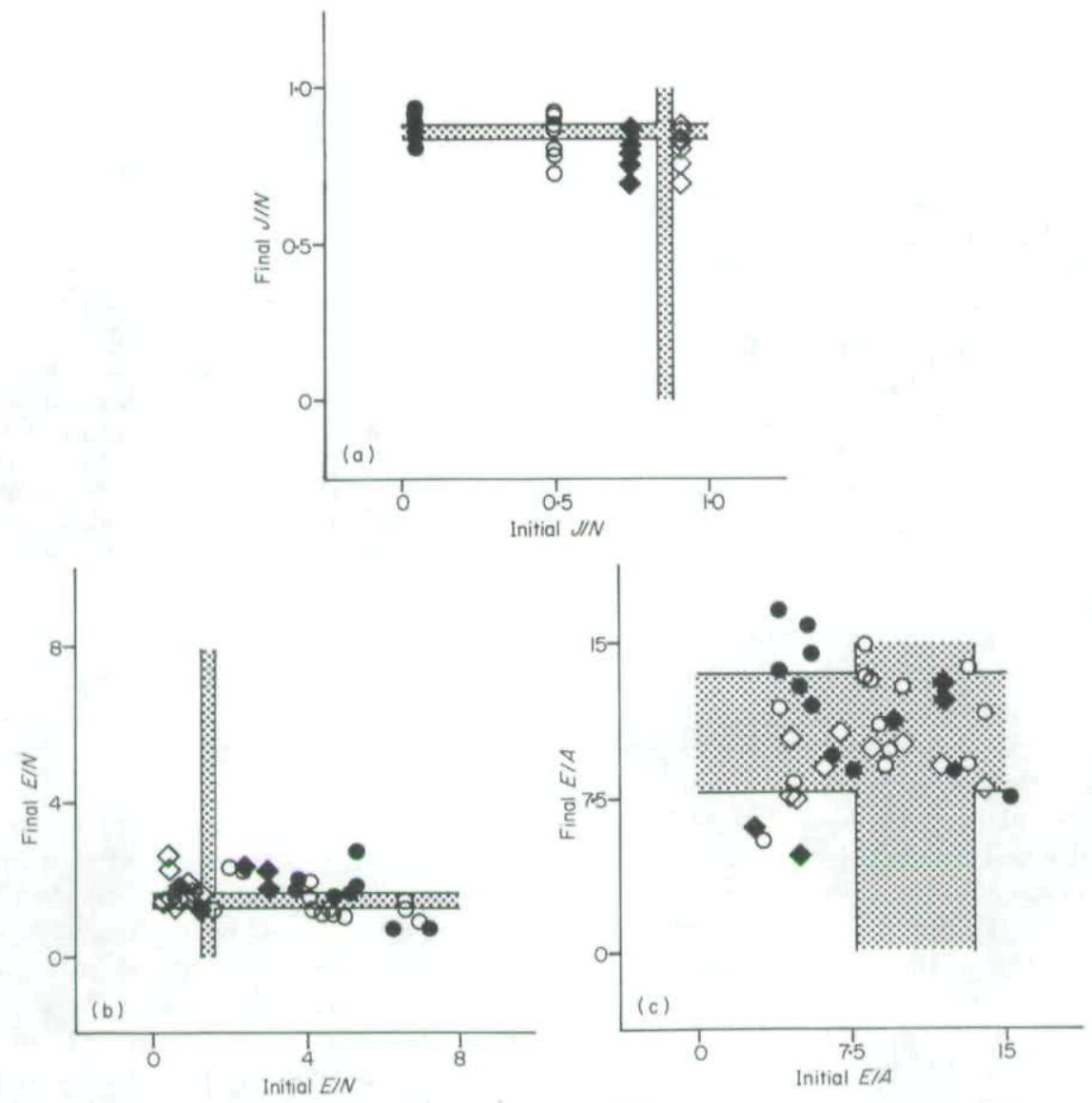

FIG. 2. Changes in structure of the experimental populations. In all three panels shaded bars show the estimated value of that measure at stable age distribution. Symbols indicate the initial proportion of juveniles ( $.0 .0 ; 0,0.50 ; 0.75 ; \diamond, 0.91$ ). (a) Initial and final proportions of juveniles $J / N$. (b) Initial and final egg ratios $E / N$. (c) Initial and final adult egg ratios $E / A$. ( $J=$ juvenile female animals; $N=$ female animals; $E=$ female eggs; $A=$ adult female animals.)

and

$$
J / N=1-e^{-b D_{J}}
$$

Equation 3 is obtained by rearranging Paloheimo's formula (method 3, Table 1); equation 4 is Taylor \& Slatkin's formula for $b$ (method 5, Table 1) and equation 5 can be derived from equations 3 and 4 . The animals used to initiate the experiments were taken from an exponentially growing population, but picked out according to size and egg number. Therefore they were not perfectly randomly distributed. We assumed, however, that the adult egg ratios $E / A$ at the end of the experiments represent the fecundity of an exponentially growing population and can be used for a rough estimation of proportions at stable age distribution. The adult egg ratio for this group of 1137 animals was $E / A=10.55$. The mean value from the thirty-seven experiments was 10.65 with a standard deviation of 2.86 . We took these values as our estimate of $E / A$ at stable age distribution and used it in equation 4 to calculate $b=0.305(+0.021,-0.027)$. Then, $E / N=1.46(+0.16,-0.19)$ was calculated from equation 3 , and $J / N=0.863$ $(+0.017,-0.027)$ was calculated from equation 5 .

The experimental populations were initiated with a wide range of proportions of juveniles. At the end of the 7-day experiments, all of the populations were close to the stable proportion 
(Fig. 2a). The populations which initially contained only adults ended with the highest range and median of the proportion of juveniles. Juveniles produced in this set of populations did not have time to mature during the experiment.

The wide initial range in the egg ratios $E / N$ (from 0.41 to 14.75 , median $=3.81$, mean $=$ $3.85, \sigma=3.13$ ) reflected the wide range in the proportion of juveniles. As the proportion of juveniles approached a stable value, so did the egg ratio (Fig. 2b). (Values of the final egg ratios ranged from 0.72 to 2.76 with median $=1.51$, mean $=1.58$, and $\sigma=0.52$.)

The adult egg ratio $E / A$ changed drastically within single experiments but the range of values was the most stable among the measures of population structure (Fig. 2c). This measure of population structure is, of course, not affected by the proportion of juveniles, although it may vary with age composition of the adult population. The initial median adult egg ratio was 8.2 ; the final median was 10.4 . As we noted above, the adults used to begin the experiments represent a sample from an exponentially growing population, and the adult egg ratio should consequently be close to the stable value. Adult egg ratios varied substantially among experimental populations because they contained small numbers of adults, whose individual fecundities were quite variable.

\section{Error in the estimated birth and death rates}

Methods for the estimating birth and death rates appeared to be biased (Fig. 3). Five of the six methods tended to overestimate death rate, with errors as large as 3.55 (Caswell) per day (Taylor \& Slatkin modified 2.46, Edmondson 1.35, Seitz 0.69, Paloheimo 0.51). Taylor \& Slatkin's method generally underestimated the death rate and showed a maximum error of -0.12 per day. Based on the median error, Taylor \& Slatkin's method was best for our data. It also showed the narrowest range of errors. Paloheimo's method was next, also its median error and range were much greater. Seitz' method gave results similar to Paloheimo's method. Caswell's method, Taylor \& Slatkin's modified method, and Edmondson's method showed substantially greater errors.
The magnitude of error depended on the starting conditions as well as on the estimation method. All of the methods performed fairly well for the initial population structure closest to the stable age structure (see Fig. 2a), $J / N=0.91$. As the proportion of adults increased, errors increased markedly for all estimates, although Taylor \& Slatkin's method showed a smaller increase in difference from expected than the others. Although Taylor \& Slatkin's modified formula was derived to compensate for departures from the stable proportion of adults in the population, it performed worst than other methods when departures from the stable proportion of adults were greatest.

\section{Simulation results}

The estimated death rate was used to measure performance of the birth rate formulae. The actual death rates in the simulations were very close to zero. The number of age classes was great enough so that the effect of mortality in the last age class was negligible. (The actual range of $d$ was $0.000-0.011$.) Death rates were calculated using three birth rate estimates and population growth rates measured over five time intervals $(1,5,10,15$ and 20 time steps, or $0.5,2.5,5,7.5$ and 10 days). These rates were calculated at each time step over the first twenty time steps of the simulations. During the first twenty time steps ( 10 days), the initial age distributions altered considerably. For example, in the simulations with increasing agespecific fecundity, at time step 0 the deviations from the stable birth rate $b=0.326$ ranged from +2.033 to +3.727 . At time step 19 the deviations ranged from +0.025 to +0.059 . For each time interval and estimation method, the first twenty estimates were combined from the simulations with each of three initial age structures (uniform, positively skewed, and negatively skewed) to calculate the results shown in Fig. 4.

As the death rates used in the simulations are zero, the ranges of the estimated death rates are the most useful indicators of the performance of these estimators (Fig. 4), because we have no measure of a typical departure from stable age distribution. The behaviour of each estimator depended strongly on the time interval over which $r$ was calcu- 

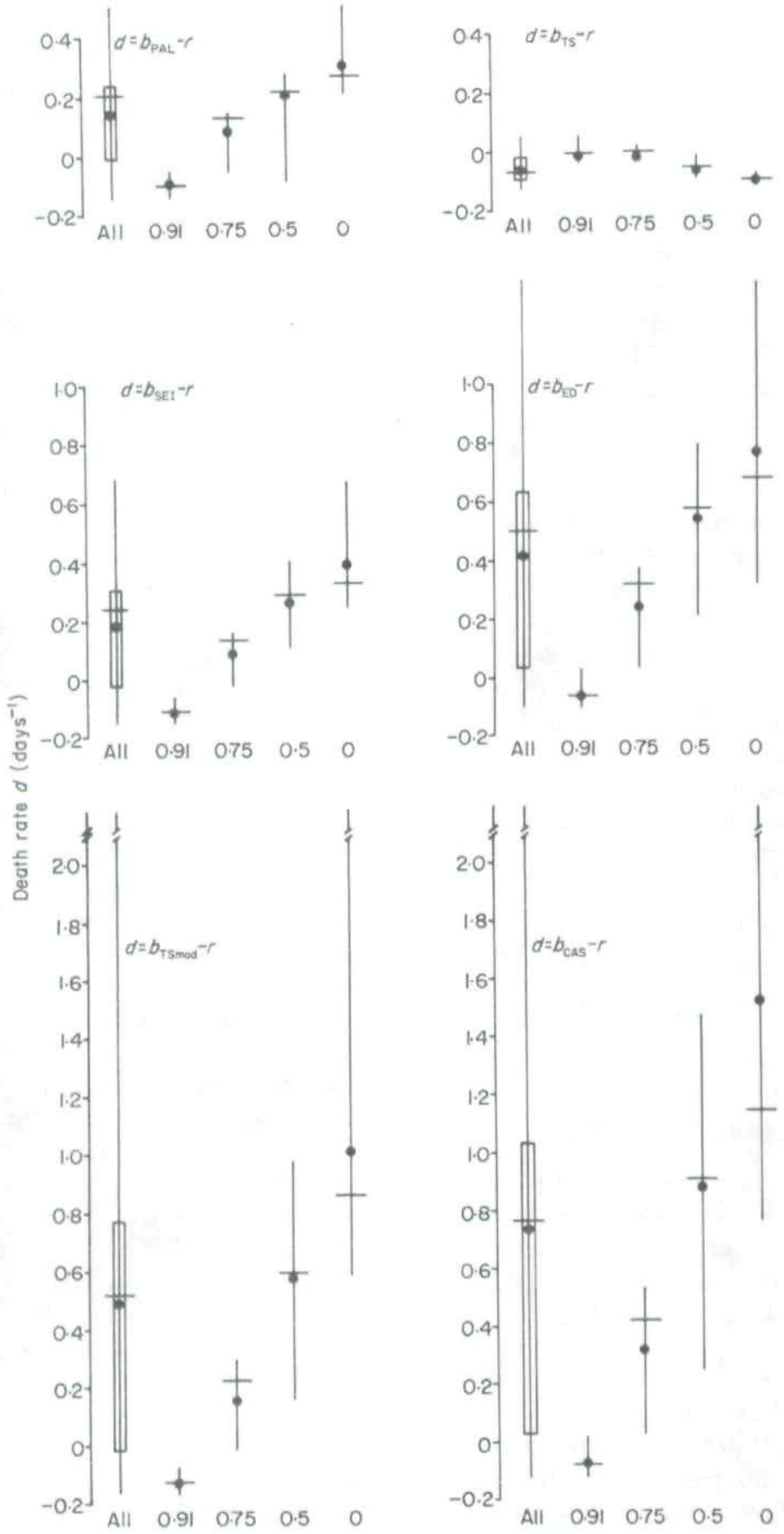

Initial proportion of juveniles $J / N$

FIG. 3. Estimated death rates $d$ for the experimental populations. Separate panels show $d$ calculated using different equations for estimating the birth rates $b$ (see Table 1 for list of equations). Within each panel data are shown for all experimental populations combined and for populations grouped by the initial proportion of juveniles $J / N$ ( $J=$ juvenile female animals; $N=$ female animals). The deviation of $d$ from 0 measures the error in the estimate of $b$.Vertical bars show ranges of values; horizontal bars show medians; circles show means. Boxes show quartiles for the combined data. 

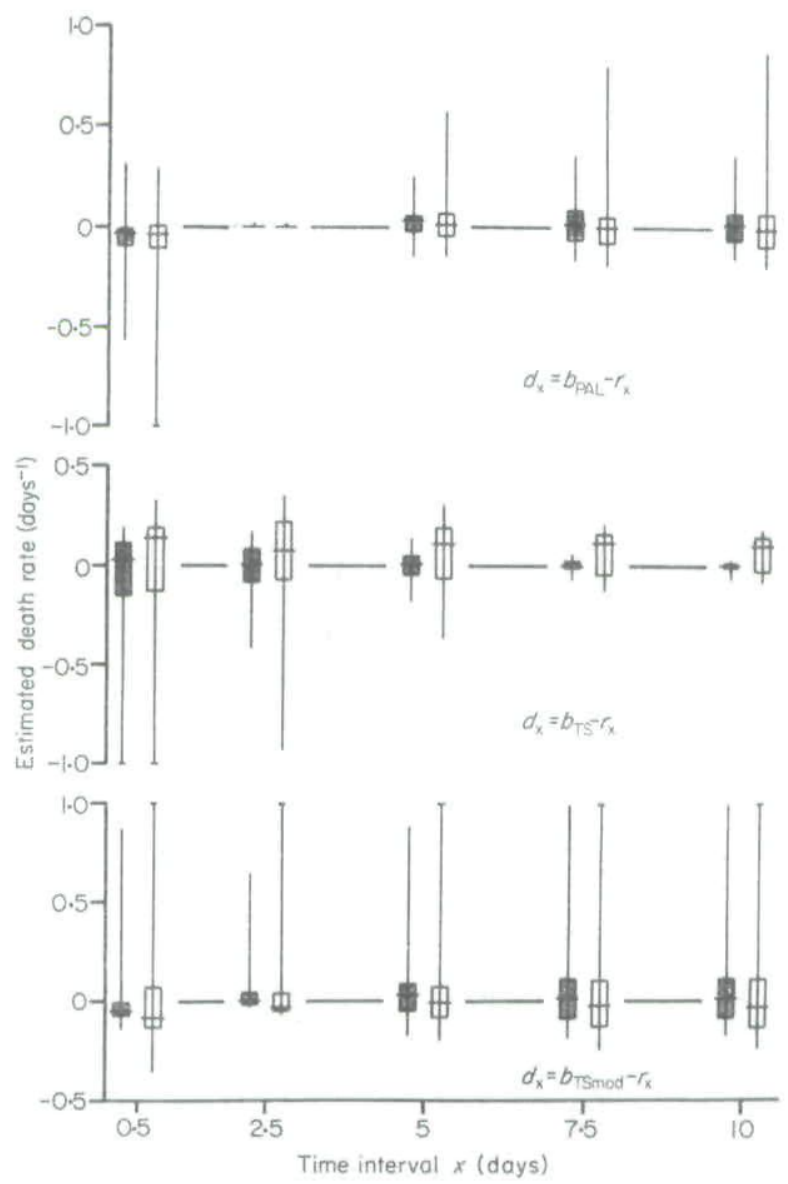

FIG. 4. Estimated death rates for the simulated popultions. Paloheimo's formula (upper panel). Taylor \& Slatkin's formula (middle panel), and Taylor \& Slatkin's modified formula (lower panel) were used to estimate the birth rates. Rates were calculated over five time intervals shown on the $x$-axis. Vertical bars show the ranges of sixty death rate estimates. Short horizontal bars indicate where ranges extend beyond the axes of the plot. Longer horizontal bars show medians, and boxes show quartiles. Shaded and unshaded boxes indicate data from simulations with constant age-specific fecundity and with increasing age-specific fecundity, respectively.

lated. At a time interval of 2.5 days (equal to the duration of the egg stage), Paloheimo's formula looks pretty good most of the time. It was substantially less reliable at shorter intervals, when it tended to underestimate rates, or at longer intervals, when it tended to overestimate them. Taylor \& Slatkin's formula produced fairly accurate estimates at longer time intervals. As judged by deviations, Taylor \& Slatkin's modified estimate was unreliable at all time intervals. Although the medians errors were generally slightly below zero, large positive deviations occurred.

The life history of the simulated population also affected the results. When fecundity increased with the age of the adult, rather than remaining constant, errors in the death rate estimates were greater. This was because the adult egg ratios varied with age structure within the adult population, magnifying the effect of the instability in age structure.

\section{Discussion}

The experiments and simulations show that unstable age structures may produce errors in estimates of birth and death rates using formu- 
lae based on egg ratios. The magnitude of error is influenced by the method chosen and the population structure. The simulations indicate that the amount of error is also influenced by the time interval over which $r$ is calculated and the animal's life history.

Over a time interval equal to the duration of the egg stage $D_{\mathrm{E}}$, Paloheimo's formula estimated the population birth rate very accurately when age-specific death rates were equal for all age classes. (The small deviations from 0 in the death rate estimates made with Paloheimo's formula and $r$ calculated over a 2.5 day interval in Fig. 4 reflect the mortality of the last age class of animals in the simulations.) All of the eggs present in the population at the time $t_{1}$ must hatch (or die) in the interval $\left[t_{1}, t_{2}=t_{1}+D_{E}\right]$. Essentially, the formula converts the proportional increment $E / N$ to the population over the interval $D_{\mathrm{E}}$ to the equivalent constant instantaneous birth rate. When population structure is changing, errors in estimates over intervals shorter than $D_{\mathrm{E}}$ occur because the birth rate is not constant over the interval. Over intervals longer than $D_{\mathrm{E}}$, changes in egg ratios also contribute to the error.

Taylor \& Slatkin's formula worked better over intervals longer than the duration of the egg stage, such as the 7-day duration of our experiments and the 7.5- and 10-day intervals in our simulations, because the average birth rate approached the stable birth rate as the population structure stabilized. If the adult egg ratio is initially close to its stable value, this method predicts what the birth rate will become as the population structure stabilizes. In the simulations it worked better for the life history with constant age-specific fecundity, because the initial adult egg ratio was unaffected by the age composition of the adult population and was consequently exactly at its stable value.

Among the methods which do not include data on the proportion of juveniles and adults Paloheimo's method was the best at the time interval used in our experiments. Seitz' method gave results very similar to Paloheimo's for the experimental populations. Because Seitz' calculations are more elaborate and require estimates of more parameters, Paloheimo's method is generally preferable.

These results suggest that Paloheimo's for- mula is the best choice for estimating birth and death rates when the interval between samples is close to the duration of egg development (see also Keen \& Nassar, 1981; Polishchuk, 1980). Both the experiments and the simulations indicate Taylor \& Slatkin's method might work better at longer sampling intervals, particularly if disturbances in the age structure of the population are episodic. Taylor \& Slatkin's modified formula seems to have no value under the conditions tested, and other methods based on direct inference of birth rates from egg age distributions are probably similarly unreliable when projected over intervals longer than some fraction of the duration of the egg stage.

Our tests simulated a single initial episode of disturbance to the age structure of a population, but the age structure will tend to stabilize after a disturbance under any conditions. The rate at which this occurs generally increases with the birth rate (not the net population growth rate). In rapidly reproducing populations, newborn juveniles quickly dominate the population structure. Because the structure changes more slowly for populations with low birth rates, Taylor \& Slatkin's formula may not be the best choice at longer time intervals. However, birth rate estimates have much smaller range of error for populations with low birth rates (Taylor \& Slatkin, 1981), and death rate estimates are consequently much more reliable; thus the choice of methods is not so critical.

Other investigators have used computer simulations to test properties of birth and death rate estimates. Seitz (1979) tested birth rate estimates using the formulae of Edmondson, Caswell and Paloheimo, and two methods that he derived. (His two-stage model is method 4 in Table 1.) He simulated population growth in a variable environment. For the two-stage models (eggs and animals; juvenile and adult animals not treated separately), his results were similar to ours. Ranked by decreasing reliability, the methods were: Seitz', Paloheimo's, Edmondson's and Caswell's. Seitz' and Paloheimo's methods gave close results. Full details of the simulations and results were not given, but he concluded that: sampling interval affected the error in the estimates; the estimates were worse when death rates were high, especially if they were 
higher for eggs and adults than for juveniles; and in general deviations from steady state conditions may produce great errors.

Polishchuk $(1980,1982)$ used computer simulations to test the methods of Leslie (1948) as modified by Polishchuk (1980), Elster (1954) and Paloheimo (1974). He found that Paloheimo's method estimated the birth rate most accurately and that all of the methods worked best at a sampling interval equal to the duration of egg development.

Differential age- or stage-specific mortality, which we did not test here, also contributes to errors in birth and death rate estimates. Other problems to consider when choosing methods and interpreting estimates include the effects of vertical stratification or migration of the population (Prepas \& Rigler, 1978; Keen, 1981) and sampling errors in estimates of parameters (DeMott, 1980; Keen \& Nassar, 1981; Taylor \& Slatkin, 1981).

The birth and death rate estimates that we have tested here are frequently used by aquatic ecologists. Error of the magnitude that our results show could significantly affect conclusions about factors affecting birth rates, especially when birth rates are high and environmental conditions are rapidly changing. It could drastically alter conclusions about processes affecting death rates. Inference about population processes that depend on these estimates must be made with caution.

As a consequence of this study we give the following practical recommendations. It is very useful to monitor the ratio of adults to juveniles. Large errors can be expected when the change in this ratio is very rapid, particularly at high birth rates. Any individual estimate of birth and death rate should be treated with caution, although a cladoceran population can achieve a near stable age distribution rather quickly following a perturbation. However, trends over several estimates or correlations based on a longer data set are more reliable (see e.g. DeMott, 1983). For general use, the Paloheimo formula is simple and reasonably accurate for slowly changing age-structures. When age-structure is changing quickly, the Taylor \& Slatkin method is most accurate provided that the juvenile developmental time is exactly known.

We hope that plankton ecologists will continue to criticize and test these methods.
Although they are an imperfect set of tools, they are among the most useful that we have for studying processes in planktonic communities.

\section{Acknowledgments}

We thank Winfried Lampert for encouraging discussions and helpful criticism. We appreciate helpful comments from Scott Cooper, William R. DeMott, Diana Engle, Mona Mort, Ace Sarnelli and Alfred Seitz.

B. E. Taylor was supported by contract DE-AC-09-76SR00819 between the United States Department of Energy and the Institute of Ecology of the University of Georgia during most of her work on this project.

\section{References}

Argentesi F., de Bernardi R. \& di Cola G. (1974) Mathematical models for the analyses of population dynamics in species with continuous recruitment. Memorie dell'Istituto di Idrobiologia, 31, 245-275.

Caswell H. (1972) On instantaneous and finite birth rates. Limnology and Oceanography, 17, 787791.

Cooper W.E. (1965) Dynamics and production of a natural population of a freshwater amphipod, Hyalella azteca. Ecological Monographs, 35, 377394.

DeMott W.R. (1980) An analysis of the precision of birth and death rate estimates for egg-bearing zooplankters. Evolution and Ecology of Zooplankton Communities (Ed. W. C. Kerfoot), pp, 337-345. University Press of New England, Hanover, New Hampshire.

DeMott W.R. (1983) Seasonal succession in a natural Daphnia assemblage. Ecological Monographs, 53, 321-340.

Dorazio R.M. \& Lehman J.T. (1983) Optimal reproductive strategies in age-structured populations of zooplankton. Freshwater Biology, 13, 157-175.

Edmondson W.T. (1960) Reproductive rates of rotifers in natural populations. Memorie dell'Istituto di Idrobiologia, 2, 21-77.

Edmondson W.T. (1968) A graphical model for evaluating the use of the egg ratio for measuring birth and death rates. Oecologia, 1, 1-37.

Edmondson W.T. \& Winberg G.G. (Eds) (1971) A Manual on Methods for the Assessment of Secondary Productivity in Fresh Waters. IBP Handbook No. 17. Blackwell Scientific Publications, Oxford.

Elster H.-J. (1954) Über die Populationsdynamik von Eudiaptomus gracile Sars und Heterocope 
borealis Fischer im Bodensee-Obersee. Archiv für Hydrobiologie, Suppl. 20, 546-614.

Gehrs C.W. \& Robertson A. (1975) Use of life tables in analysing and the dynamics of copepod populations. Ecology, 56, 665-672.

Hairston, N.G., Jr \& Twombly S. (1985) Obtaining life table data from cohort analyses: a critique of current methods. Limnology and Oceanography, 30, 886-893.

Hall D.J. (1964) An experimental approach to the dynamics of a natural population of Daphnia galeata mendotae. Ecology, 45, 94-112.

Haney J.F. \& Hall D.J. (1973) Sugar coated Daphnia: a preservation technique for Cladocera. Limnology and Oceanography, 18, 331-333.

Keen R. (1981) Vertical migration, hatching rates, and distribution of egg stages in freshwater zooplankton. Journal of Thermal Biology, 6, 349-351.

Keen R. \& Nassar R. (1981) Confidence intervals for birth and death rates estimated with the egg-ratio technique for natural populations of zooplankton. Limnology and Oceanography, 26. $131-142$.

Keyfitz N. (1977) Introduction to the Mathematics of Population, with Revisions. Addison-Wesley Publishing Company, Reading, Mass.

Kirsch S. (1982) Untersuchungen zum Einfluß von Störfaktoren auf die Aussagekraft populationsdynamischer Modelle: Test mit Laborpopulationen von Daphnia pulicaria. Diplom-Theses, Gōttingen.

Lampert W. (1975) A laboratory system for the cultivation of large numbers of Daphnia under controlled conditions. Archiv für Hydrobiologie, 48, 138-140.

Leslie P.H. (1948) Some further notes on the use of matrices in population mathematics. Biometrika, 35, 213-245.

Lynch M. (1982) How well does the EdmondsonPaloheimo model approximate instantaneous birth rates? Ecology, 63, 12-18.

Lynch M. (1983) Estimation of size-specific mortality rates in zooplankton populations by periodic sampling. Limnology and Oceanography, 28, 533-545.

Muck P. \& Lampert W. (1980) Feeding of fresh water filter-feeders at very low food concentrations: Poor evidence for 'threshold feeding' and 'optimal foraging' in Daphnia longispina and Eudiaptomus gracilis. Journal of Plankton Research, 2, 367-379.

Paloheimo J.E. (1974) Calculation of instantaneous birth rates. Limnology and Oceanography, 19, 692-694.
Polishchuk L.V. (1980) A comparison of different methods of estimation of birth and death rates in plankton animals. Zhurnal Obshchei Biologii, 41, 125-137 [in Russian with English summary].

Polishchuk L.V. (1982) The 'negative mortality' of planktonic animals and sampling schedule. Zhurnal Obshchei Biologii, 43, 411-418 [in Russian with English summary].

Polishchuk L.V. \& Ghilarov A.M. (1981) Comparison of two approaches used to calculate zooplankton mortality. Limnology and Oceanography, 26, 1162-1168.

Pollard, J.H. (1973) Mathematical Models for the Growth of Human Populations. Cambridge University Press.

Prepas E. \& Rigler F.H. (1978) The enigma of Daphnia death rates. Limnology and Oceanography, 19, 970-988.

Ricker W.E. (1975) Computation and interpretation of biological statistics of fish populations. Fisheries Research Board of Canada, Bulletin, 191.

Rigler F.H. \& Cooley J.M. (1974) The use of field data to derive population statistics of multivoltine copepods. Limnology and Oceanography, 19, 636-655.

Rigler F.H. \& Downing J.A. (1984) The calculation of secondary productivity. A Manual on Methods for the Assessment of Secondary Productivity in Fresh Waters (Eds F. H. Rigler and J. A. Downing), 2nd edn, pp. 19-58. IBP Handbook No. 17. Blackwell Scientific Publications, Oxford.

Romanovski Y.E. (1984) Prolongation of postembryonic development in experimental and natural cladoceran populations. Internationale Revue der Gesamten Hydrobiologie, 69, 149-157.

Seitz A. (1979) On calculation of birth rates and death rates in fluctuating populations with continuous recruitment. Oecologia, 41, 343-359.

Taylor B.E. \& Slatkin M. (1981) Estimating birth and death rates of zooplankton. Limnology and Oceanography, 26, 143-158.

Threlkeld S.T. (1979) Estimating cladoceran birth rates: the importance of egg mortality and the egg age distribution. Limnology and Oceanography, 24, 601-612.

Winberg G.G. (1971) Methods for the Estimation of Production of Aquatic Animals (Transl. by A. Duncan). Academic Press, London.

Wright I.C. (1965) The population dynamics and production of Daphnia in Canyon Ferry Reservoir, Montana. Limnology and Oceanography, 10, 585-590.

(Manuscript accepted 6 April 1987) 
Copyright of Freshwater Biology is the property of Blackwell Publishing Limited and its content may not be copied or emailed to multiple sites or posted to a listserv without the copyright holder's express written permission. However, users may print, download, or email articles for individual use. 